Wetering, R. van de, Batenburg, R. A PACS maturity model: a systematic meta-analytic review on maturation and evolvability of PACS in the hospital enterprise. International Journal of Medical Informatics: 2009, 70t2 127-140

\begin{tabular}{|l|l|}
\hline $\begin{array}{l}\text { Postprint } \\
\text { Version }\end{array}$ & 1.0 \\
\hline Journal website & $\underline{\text { http://dx.doi.org/10.1016/j.ijmedinf.2008.06.010 }}$ \\
\hline Pubmed link & $\underline{\text { http://www.ncbi.nlm.nih.gov/pubmed/18752988 }}$ \\
\hline DOI & $10.1016 /$ j.ijmedinf.2008.06.010 \\
\hline
\end{tabular}

This is a NIVEL certified Post Print, more info at http://www.nivel.eu

\title{
A PACS maturity model: A systematic meta-analytic review on maturation and evolvability of PACS in the hospital enterprise
}

\author{
ROGIER VAN DE WETERING ${ }^{\mathrm{A}}$, AND RONALD BATENBURG ${ }^{\mathrm{A}}$, \\ ${ }^{a}$ Department of Information and Computing Sciences, Utrecht University, P.O. Box 80.089, 3508 TB \\ Utrecht, The Netherlands
}

\begin{abstract}
Introduction: With PACS and medical imaging technology maturing, the importance of organizational maturity and effective deployment of PACS in the hospital enterprise are becoming significant.

Objective: The objective of this paper is twofold. Firstly, PACS literature on maturity and evolvability in the hospital enterprise is analyzed, resulting in an overview of the relevant developments concerning maturity of PACS. Secondly, this paper looks at the development of a maturity model for PACS technology.

Methods and results: Using structured search queries, we identified 34 papers reporting relevant aspects of maturity and evolvability of PACS. From the results of a meta-analytic review on PACS maturity and evolvability, we propose a model - the PACS maturity model (PMM) - that describes five levels of PACS maturity and the corresponding process focus.

Conclusion: We argue that this model can help hospitals to gain insights into their (strategic) objectives for growth and maturity with regard to PACS, the electronic patient record (EPR) and other health information systems. Moreover, the proposed model can be applied as a valuable tool for organizational assessments, monitoring and benchmarking purposes. Hence, the PMM contributes to an integral alignment model for PACS technology.
\end{abstract}

\section{INTRODUCTION}

The field of healthcare and medical informatics has gradually evolved through a number of stages and established itself in recent years. The field is very broad and includes advances that have been made in medical imaging technology, e.g. picture archiving and communication systems (PACS), information systems, image-guided surgery and therapy, computer-aided diagnosis (CAD), decision-support systems and the electronic patient record (EPR) [1], [2] and [3]. The field is still evolving although some claim that the area of PACS has matured and is no longer cutting-edge [4] and [5]. Others claim that PACS has been introduced into clinical practice too soon, and with too much hype, and research and development are very much in need now [6]. The utilization of the rapidly-growing results and possibilities of non-invasive digital imaging systems in clinical application, and related research and developing work, are also serious challenges [6] and [7]. Moreover, Hood and Scott [8] mention that to date there is little published information concerning the clinical impact of PACS in the working environment. 
Wetering, R. van de, Batenburg, R. A PACS maturity model: a systematic meta-analytic review on maturation and evolvability of PACS in the hospital enterprise. International Journal of Medical Informatics: 2009 7062 127-140

The concept of picture archiving and communication systems (PACS) was introduced as early as 1982 and after more than 20 years of research, development and implementations, PACS has become an integrated component of today's healthcare delivery system [9]. PACS can therefore be considered as the fundamental infrastructure for digital diagnostic imaging and information management systems. PACS originated as an image management system for improvement for the efficiency of radiology practice and evolved into a hospital-integrated system dealing with multimedial information. The integration of many different types of information requires the technology of multimedia: hardware platforms, information systems and databases, communication protocols, display technology and system interfacing and integration [10].

There are many definitions for PACS, ranging from simple IS/IT used for digitizing images to enterprisewide image management systems and integrated workflow systems that streamline all operations throughout the whole patient-care delivery process. For instance, Huang [4] defines PACS as a workflowintegrated imaging system that is designed to streamline operations throughout the entire patient-care delivery process. Anderson and Flynn [11] did a systematic literature review of a broad range of topics about PACS. They defined PACS more from a technical point of view: picture archiving and communication systems (PACS) are high-speed, graphical, computer network systems for the storage, retrieval and display of radiological images. As the definitions indicate, PACS is a very broad term encompassing many related, but different, components and systems related to medical imaging for clinical practices [12]. PACS can thus be both very simple and be a more complex enterprise-wide system.

Although PACS is now a well-established technology, achieving a filmless environment with PACS is still a high-cost venture [13]. A successful method for implementing and aligning PACS in the hospital enterprise would therefore be a prerequisite, and insight into the current and desired level of maturity of PACS valuable to the hospital.

So, how can PACS maturity be modelled, measured and assessed, and what is known from current research on this fundamental topic in medical informatics?

Theories on information systems and information technology (IS/IT) maturity and adoption are wellestablished in business and IS/IT literature going back to the early 70s. The concept of the stage hypothesis was introduced by Nolan [14] in 1973, extended [15] and frequently discussed and adapted [16], [17], [18], [19] and [20]. In general, the IS/IT maturity models provide insight into the structure of elements that represent process effectiveness of IS/IT in organizations [21].

In this research, we develop a model that can be used to assess the alignment and maturity of PACS, and PACS deployment performance within hospital enterprises. Since PACS is a system designed to streamline operations through the entire patient-care delivery system, one would expect that it makes a significant difference in terms of throughput and clinical action as well. It is suggested that theories on Business/ITalignment, organizational fit and adoption of IS/IT can help us to understand why certain key elements in clinical practice have not been [22] achieved. In a first step to construct an integrative implementation/alignment framework for PACS, insight into the levels of maturity of PACS is a prerequisite. Next to the organizational aspects [23] and [24] the framework should enable the quantification of PACS maturity including its relation to PACS deployment performance. In this paper, we present the results of an extensive and systematic literature search that is performed in order to construct such a model that sets out PACS maturity and evolvability in the hospital enterprise.

To the best of our knowledge, we are the first in this as so far there is no reference to a recognized maturity model for PACS, and thus no valuable tool for empirical research.

\subsection{Outline of the paper}

The next section reviews the history of the PACS and sets out the relevant developments concerning IS/IT maturity and the maturity of hospital information systems. The method for our structured literature search is detailed in Section 3. Section 4 presents the review of maturity and evolvability of PACS literature and Section 5 proposes growth levels for PACS and a PACS maturity model based on a meta-analytic approach. Finally, we discuss both the opportunities and limitations of this study and the constructed PACS maturity model (Section 6). 
Wetering, R. van de, Batenburg, R. A PACS maturity model: a systematic meta-analytic review on maturation and evolvability of PACS in the hospital enterprise. International Journal of Medical Informatics: 2009 10.2 127-140

\section{BACKGROUND}

\subsection{PACS history}

The concept of a PACS was introduced more than two decades ago and the desire to store medical images digitally stems from well-known limitations in the film-based radiology departments [25]. The initial development of diagnostic imaging started over 50 years ago with the utilization of image-intensifier TV systems for fluoroscopy and the development of gamma camera for radionuclide imaging. Over the years much research has been done on electronic imaging, MRI, PET, SPECT, the development of computed tomography (CT), ultrasound imaging and PACS [26].

A PACS acquires medical images digitally from several modalities in the radiology department (e.g. CT, Ultrasound, MRI, plain X-ray), stores them in central data repositories/databases and makes them available upon request by, for instance, referring clinicians. Since the medical images are now in browser-based format, they can be made available for viewing almost instantaneously throughout the entire hospital and even beyond the boundaries of hospitals to off-site radiologists and other institutions using secure broadband internet connections.

Nowadays the PACS industry is a well-matured industry and offers archiving solutions and reading stations that fulfil the needs of the users [27]. The Integrating the Healthcare Enterprise [28] also provides hospitals with a solid framework that from a technical point of view ensures that different information systems - including HIS, RIS, EMR - are well integrated. PACS are deployed in most academic centres and many private practices are joining the ranks of the digital radiology revolution [5]. A recent survey on E-business in 2006 among 18 European countries, conducted by Empirica by order of the European Commission, posed the basic question for hospitals as to whether they use PACS or not. The (unweighted) result of this enquiry is presented in Fig. 1. Large differences between countries appear from these data. Only $10 \%$ of French hospitals say they use PACS as against almost $70 \%$ of Finnish hospitals. It is difficult to discover a definite systematic adoption pattern within this list of countries in terms of their geographical location, economic position, healthcare system or ICT usage. This remains an interesting topic for further research. At this point, it can be noted that among a large number of European countries the use of PACS in hospitals is at a certain level but is still - given the European average of $33 \%$ - in its growing stage.

\section{[FIGURE 1]}

The highest rate of adoption is seen in the Scandinavian countries of Norway and Finland. This comes as no surprise, since Scandinavia leads the global industry with the highest average adoption rates of digital solutions in healthcare.

Some scholars consider PACS to be a mature technology, but not all of the key issues have been resolved [5]. For instance, there is a wide variety in features and capabilities of software products. The systems are complex, and costly to acquire, replace, maintain and repair. The performance of these systems directly affects patient care and (clinical) workflow and clinical effectiveness in the working environment [8]. Samei et al. [5] therefore suggest that careful attention should be paid to the selection of a system that meets certain needs and requirements. With recent developments in terms of integration of PACS with other technologies and add-ins like chat and messenger services and emailing functions [29], PACS still requires attention. Moreover, utilization of the rapidly-growing results and possibilities of non-invasive digital imaging systems in clinical application and related research and developing work, are serious challenges [7].

\subsection{Information system maturity}

The concept of IS/IT maturity and adoption is well-known in business literature and goes back to the early 70s. Within the field of information systems Nolan and Gibson [14] and [15] are considered to be the founders of the IS/IT maturity perspective. From research on the usage of IS/IT in large US organizations, they proposed an evolutionary model initially containing four stages of growth. Later, two more stages were added to the initial model. The stages are: initiation, contagion, control, integration, data management and maturity. The six-stage model represents the level of IS/IT expenses for an organization in relation to the stages of increasing sophistication and maturity of the IS/IT. According to this model, IT-adoption or IT-management will see gradual uptake by a relatively small group (of organizations) at the beginning. This adoption phase is subsequently followed by a large group, and finally by a smaller group that stays behind 
Wetering, R. van de, Batenburg, R. A PACS maturity model: a systematic meta-analytic review on maturation and evolvability of PACS in the hospital enterprise. International Journal of Medical Informatics: 2009 7062 127-140

in terms of adoption of emerging technologies. In general, this pattern of adoption is called an S-curve, since it bears resemblance to a cumulative frequency distribution of adoption within different groups [30].

First published in 1989 by Watts Humphrey, and later by the Software Engineering Institute (SEI) at Carnegie Mellon, the Capability Maturity Model (CMM) - later superseded by the CMM integrated - has become an established model in the field of information systems development. CMM has been established by SEI to help organizations enhance and boost their software processes and has been recognized as a standard maturity model.

The CMM provides software organizations with guidance on how to gain control of their processes. It can help improve the maturity of these processes (of developing and maintaining software). The latest version of CMM is the CMMI (Capability Maturity Model Integration). To exemplify a staged representation five designated levels have been defined (see Table 1). Each maturity level is a defined evolutionary plateau of process improvement and includes a checklist to evolve on to the next level [31].

\section{[TABLE 1.]}

As De Bruin [32] sees it, the CMMI model is an improvement model as it provides a roadmap to get to the next level of maturity. In a recent meta-analysis on the effects of the CMM, Galin and Avrahami [33] were only able to identify three studies that give details on productivity gains when an organization progresses to CMM level 2 and only 12 studies that provide details on productivity gains when an organization progresses to CMM level 3.

In general, the IS/IT maturity models provide insight into the structure of elements that represent process effectiveness of IS/IT in organizations [21]. The concept of defining stages of development and growth has been further extended and applied to organizations. Examples are the stage maturity model which Holland and Light [34] developed for enterprise resource planning (ERP) systems and a stage model for Intranet implementation by Damsgaard and Scheepers [35].

Health information systems (HIS) have been around for more than two decades, and tremendous progress in medicine as well as informatics has been realized since then [36]. Haux identifies several lines of development for HIS:

- the shift towards computer-based processing and storage, as well as the increase of data processing;

- the shift from local to global information system architectures;

- besides professional use of the HIS, that of patients and health consumers as well;

- the usages of the data not only for patient care and administrative purposes, but also for healthcare planning and clinical research;

- the shift of focus from mainly technical HIS problems to those of change management as well as of strategic information management;

- a shift from (predominantly) alpha-numerical data to clinical images and even now also to data on the molecular level;

- the steady increase of new technologies to be included in order to enable continuous monitoring of the health status of patients.

Similar development stages can be found in Vogel [37], who outlines several phases of investments in IT in healthcare. With each phase of IT investment in healthcare greater expectations and more complex systems environments are produced in which subsequent IT-investments are being made.

\section{METHODS}

\subsection{Structured literature search}

As a starting-point, the review of the literature employed search queries using keywords in OMEGA, the central search engine of Utrecht University containing thousands of digital journals from different disciplines and accessing over 13 billion full-text papers. Publishers included are Ebsco, Elsevier Science Direct, JStor, SpringerLink, IoP, ArXiv, Karger, Pubmed Central, Project Muse and DOAJ Lund. A literature search was also performed by means of Scholar (Google).

The following structured queries were performed:

- PACS, digital radiology, diagnostic imaging, + \{maturity | evolution | progression | stages | growth | development 
Wetering, R. van de, Batenburg, R. A PACS maturity model: a systematic meta-analytic review on maturatiog and evolvability of PACS in the hospital enterprise. International Journal of Medical Informatics: 2009 706 127-140

The queries resulted in a long list of retrieved scientific papers, of which in total 131 concern the PACS domain. Subsequently, inclusion criteria relevant to a particular PACS study were determined based on their theoretical and practical value for our research purpose rather than statistical validity. These papers were then screened for relevance for the purpose of this investigation by use of the following criteria:

- Elaboration on the application of PACS other than the basic PACS functionality (acquisition, storage, distribution and display);

- Focus on the usage and application process of PACS in the hospital, not merely on PACS technology and outcome;

- Description of the evolutionary/maturity process of PACS in the hospital (enterprise);

- The study is empirically applied, tested and/or validated in hospitals and clinics (health systems);

- The study mentions organizational aspects relevant to the application of PACS in the hospital.

All the papers retrieved through structured queries were manually matched against the above inclusion criteria. After the application of these criteria, the total of papers to be reviewed was 25 references. To enhance the reliability of our applied research strategy, and to make sure that all relevant papers were included for our review purpose, a second researcher performed the above queries. After manual matching of the inclusion criteria another two papers [38] and [39] were retrieved.

These papers were retrieved with a structured query, and several other papers were selected from main publications and positioning papers referring to several important publications and researches on different aspects of PACS usage. This resulted in the inclusion of another seven papers and books [9], [10], [40], [41], [42], [43] and [44] for review in this study. In the end, 34 papers were selected through the above search strategy and were input for our literature review for PACS maturity and evolvability. All papers included in the study were published between 1996 and 2007.The review method applied to the selected papers is described in Sections Sections 4 and 5.

\subsection{Qualitative meta-analysis}

A qualitative meta-analysis approach [45] and [46] is performed in order to analyse the literature, define maturity levels for PACS and construct a model based on the reviewed literature. Standard meta-analysis commonly compares and combines quantitative studies in order to obtain generalizable outcomes [47]. Its counterpart, qualitative meta-analysis, is less matured, although the method is gaining in popularity and there is a growing body of knowledge of this qualitative method [48], [49] and [50]. Here, we apply qualitative meta-analysis by three distinct analytical phases: (1) meta-theory; (2) meta-method; and (3) meta-data-analysis [45] and [51]. These three steps form the basis for the process whereby new theorization can be generated [52].

For the meta-data-analysis phase, we adapted the approach for qualitative meta-analysis as presented in the metaethnography from Noblit and Hare [45] and a study by McCormick et al. [49]. As the first step, common ground and interest for research purposes were created by exploration of the PACS literature. We then retrieved many studies for analysis purposes (see method, Section 3). After that, the studies were read iteratively. As the synthesis developed, studies were read and reread to check all the relevant topics and interpretations. This resulted in an overview of the relevant literature concerning the trends in PACS maturity and evolvability (see Section 4). Subsequently, PACS process focuses were identified and structured according to initial stages of maturity resulting in a schematic overview of PACS process focus and initial structure for maturity levels. In our quest for a maturity model, we raised questions, reread studies in order to enrich the current interpretations of the study and synthesize them. This step in the metaanalysis resulted in an ordered schema of maturity levels with the associated process focus and scientific references. Next, we related the maturity levels and tried to identify relationships and dependencies. This was done iteratively by skipping back-and-forth between the different analytic steps in the process. This fundamental step in the analysis process allowed us to achieve full richness and understand the complexity of the data. The application of this back-and-forth method in the final step provided interpretation for the constructed model while preserving the original meaning and outcomes of the studies that were involved.

These phases and steps in the meta-analysis-process enabled us to gain a comprehensive understanding of PACS literature from a maturity and evolvability perspective. This does not imply that we have created a 'new truth' concerning PACS, rather we claim to reflect on the current PACS literature in a new way. The main result of this will be an integrated maturity model for PACS based on meta-analytic grounds, as will be presented below. 
Wetering, R. van de, Batenburg, R. A PACS maturity model: a systematic meta-analytic review on maturatiog and evolvability of PACS in the hospital enterprise. International Journal of Medical Informatics: 2009 706 127-140

\section{TRENDS IN PACS MATURITY AND EVOLVABILITY}

Our systematic literature study sets out theoretical and empirical contributions in order to provide an overview of the maturity levels of PACS technology in the hospital enterprise. As a result of the metaanalysis, the synthesis provided a new overview of the relevant literature. More specific, we identified three trends of PACS maturity and evolvability:

1. Radiological and hospital-wide process improvements;

2. Integration optimization and innovation;

3. Enterprise PACS and the electronic patient record (EPR).

The retrieved articles (see Section 3) and other base resources are structured and presented in the following subsections.

\subsection{Trend 1: Radiological and hospital-wide process improvements}

\subsubsection{PACS technologies}

Using PACS, hospitals can distribute imaging data using web technologies and web servers to access, view and even manipulate PACS images in an intranet and internet environment. Furthermore, components ${ }^{1}$ architecture and technologies have a predominance in high performance primary diagnostic imaging display workstations [53]. A combination of both distribution methods allows for access, viewing and manipulation of imaging data more simply than by use of display workstations.

The integration of PACS in a filmless radiology environment results in less circulation of paper work, and that enhances the reliability of the reports [54] and [55]. Because of its focus on medical images, however, PACS is restrictive in managing (hospital) workflows. The evolution of PACS towards a system that can handle workflow management requires important alterations [42]: the emphasis should be less on images and more on multimedia documents. Software for viewing images has to coexist with administrative tools on diagnostic workstations. More advanced usage of PACS supports patient folder management by event trigger mechanisms [10]. The PACS controller and archiving server manages the patient studies in folders and contains all relevant images, reports and impressions. A folder remains online at workstation during the patient's stay or visit. Such a patient folder can be seen as the prelude to an electronic patient record (EPR), only with a focus on imaging. Main modules of a folder are archive management, network management and display/server management.

$\mathrm{Li}$ et al. [56] did an analysis on the evolution of display technologies in PACS applications and (clinical) workflow and distinguished three phases:

1. During phase 1, the focus was centred on the individual hospital department and optimized the ability to display on a stand-alone workstation;

2. In phase 2, the focus shifted towards clinical and enterprise workflow and image viewing workflow was optimized for workstation presentation;

3. For the last phase - the modern period - standardisation and enterprise integration have become key words where institutional/regional enterprise level, multi-vendor and PACS/RIS integration have become main themes.

\subsubsection{PACS implementation}

One of the major drawbacks of early PACS implementations was the proprietary, vendor-specific design of image and data communication between individual PACS components [25]. With the early PACS implementations, hanging protocols on workstations were primitive, lacking user control over series placement, window/level settings, and the zoom factor and panning required [57]. In principle, the early PACS implementations focused primarily on providing the basic PACS functions (image retrieval, viewing) and often even failed to do that [25]. These issues have been overcome by second generation PACS implementations that favour a gradual implementation in the field where it is technically feasible, economically possible and contributes to medical knowledge [58], [59] and [60].

\subsubsection{Workflow consequences}

Although workflow changes are apparent, Siegel and Reiner [61] suggest that the transition to filmless operation alone results in a relatively small gain in productivity if it is not accompanied by a redesign of the basic departmental workflow. This requires a high level of integration of the various imaging information 
Wetering, R. van de, Batenburg, R. A PACS maturity model: a systematic meta-analytic review on maturatiog and evolvability of PACS in the hospital enterprise. International Journal of Medical Informatics: 2009 7062 127-140

systems and HIS and RIS. Historically, PACS runs on a separate patient database, which may not match the implemented RIS database within a hospital. If this is the case, PACS may not contain information about exams scheduled in the RIS entity, making efficient prefetching and distribution of imaging data impossible [25]. Initially, within radiology hard-copy films were replaced by soft-copy images, although navigation remained relatively static. In this phase, many radiologists did not take full advantage of the workflow and interpretation opportunities available. Workflow enhancement can be seen in the usage of dynamic softcopy reading and stack modes further enabling the ability quickly and efficiently to display comparison studies using automated hanging protocols and allowing radiologists to navigate rapidly through crosssectional imaging data sets (using cine loops). This evolution continued towards more complex and advanced techniques that can process large volumetric data sets into two- and three-dimensional reconstructions (for further review see [60]). Crowe and Sim [62] describe a RIS/PACS implementation that is considered to be of assistance in clinical decision-making in a wide variety of clinical areas and patient management, and this is consistent with other studies. See, for instance [7]. PACS therefore not only provides workflow transformation within the hospital enterprise, it also assists in the clinical process of diagnosis and decision-making.

To describe the evolution of workflow in radiology departments, Fu et al. [63] identified four application levels.

1. The first level is the traditional environment that is based on handwriting paper and printed films;

2. The second level is a mixed and transitional radiology environment, where radiologists input text in a computer, but still read traditional hardcopy films on light boxes and use independent RIS systems;

3. At the third application level, Fu describes the true soft reporting and verification systems - even based on digital high resolution four-screen portrait monitors and/or dual projectors with two large screens, which have achieved satisfactory effects;

4. At level 4 there is an integrated digital consulting and teaching environment based on satellite and webbased technology for telemedicine, teleradiology and medical education (see also [7] and [10]).

Clearly, the initial stages are more focused on the infrastructure and the latter are more oriented towards efficient PACS workflow, process integration and alignment within the hospital. Efficient PACS workflow in an integrated healthcare IT environment and quality assurance/control procedure are generally omitted, however [63].

\subsection{Trend 2: Integration optimization and innovation}

\subsubsection{Integration}

During the development and the initial implementations of PACS and digital imaging network in hospitals, signs of PACS evolution and the possibilities for new developments and applications were clearly visible [39]. For instance, next to the development of advanced workstations and distributed medical networks, the clinical value of integrating imaging data with other image-related data into a medical record was also seen to result in adequate patient care. The rise of the internet and the extension of computerized medical records and the accessibility to other relevant clinical and image-related data through the use of multimodality workstations were recognized to have a significant impact on clinicians' and physicians' work.

By integration of PACS with the radiology information system (RIS) and other information systems in radiology, it is possible to recover statistical information allowing for a quantitative control mechanism. According to [38], this will help detect critical points in the hospital service workflow. Moreover, by integration of PACS with the HIS and RIS, the diagnosis process can be optimized, since clinical diagnosis, radiological reports and patient history are necessary at the PACS workstation to complement the imaging data at hand. The integration of the HIS, RIS and PACS image data and all related text information acquired can be managed in such a way that it is useful to a radiologist during the diagnostic process [10].

As an example of integration, PACS can optimally support the non-clinical application of the imaging system by providing electronic teaching files conforming to new MIRC (Medical Image Resource Center) standards [64]. Using this process design, hospitals can use PACS for close collaborations in teaching and research. Moreover, the construction of a medical content repository for teaching files and e-learning can function as a knowledge management system [65]. The integration of medical informatics and online learning repositories (e-learning) can be employed in many advanced applications, such as knowledge management, telemedicine, home-care, etc. Since PACS contains large amounts of imaging data, Huang [9] 
Wetering, R. van de, Batenburg, R. A PACS maturity model: a systematic meta-analytic review on maturation and evolvability of PACS in the hospital enterprise. International Journal of Medical Informatics: 2009 7062 127-140

argues that advantage can be taken of this valuable resource through the investigation of innovative clinical service, research and education using the concept of imaging informatics. Moreover, a PACS implementation allowing simultaneous presentation and post-processing capabilities of medical images plays a central role in making quick and reliable clinical decisions non-invasively and is effective in the diverse fields of consultation, education, tele-evaluation and post-processing [7].

\subsubsection{Technological advances}

With the trends towards PACS integration optimization many technological advances are made, and some emerging technologies such as computer-assisted readings (CAR) and computer-aided diagnosis can be incorporated [8]. Not only should PACS be able to incorporate new technologies/advances in terms of technical requirements, PACS can also act as a central image provider for post-processing and workflow of clinical and research medical images. As medical imaging became crucial to other departments outside radiology (for instance in the diffusion and perfusion MR imaging for stroke detection in neurology), PACS has become a clinical collaboration tool. In this respect, some call PACS an "inter-disciplinary tool" [66].

To sum up, technological advances with PACS and examples of how this is applied in clinical collaboration include the following:

- Computer-assisted bone age assessment that can be integrated with clinical PACS for advanced image analysis purposes. In PACS, a CAD (computer-aided diagnosis) based on phalangeal and carpal bone growth can be developed to assess the bone age [9], [10] and [67];

- Computer-assisted/aided diagnosis (CAD) and cuing and the adoption of intelligent application of informatics [43]. The adoption of CAD will become routine in the coming years, especially in the detection of lung nodules and breast cancers;

- Full field digital mammography (FFDM) benefits of computer-aided detection, computer-assisted classification and PACS can be realized [40]. The integration and application of breast imaging and PACS are associated with several challenges with respect to (soft copy) image display and interpretation, communication and storage, since FFDM requires high resolution monitors to enable the physician to visualize the minute details for accurate diagnosis;

- Three-dimensional (3D) imaging services as well as the workflow involved in integration of 3D imaging services with PACS and radiology information systems are serious challenges for hospitals [41];

- Image-assisted surgery system (IASS) is designed on the concept of an image-integrated EPR and use of existing knowledge of PACS and the associated medical imaging informatics infrastructure [10]. IASS allows patient-centred images and data to be staged at the server and delivered to the workstation for review before, during and after surgery;

- Intelligent data-mining will also take on greater importance with PACS [60]. It will allow for image processing and data extraction that will ease the interpretation process for radiologists even before the current data sets are presented to them. Mining objects include (radiology) reports, correlative imaging studies and electronic medical records.

\subsection{Trend 3: Enterprise PACS and electronic patient record (EPR)}

Owing to data exchange standards (HL7/DICOM), PACS can be integrated with Departmental IS and the HIS and this provides a basis for a communication platform in medicine, constituting an electronic patient record (EPR) The EPR supports an inter-disciplinary treatment of patients by providing data of findings and images from clinics treating the same patient [68]. While the PACS evolution mainly focused on imaging data and integration with RIS, the parallel evolution of electronic medical records supporting all the other clinical electronic documents led to the integration of imaging data with the rest of the patient record [69]. This integration improves the accuracy and efficiency of patient management and is enabled by the functional integration of medical data from different sources. Moreover, the experience of many practitioners shows that it is essential to integrate PACS imaging data in the patient's medical record in order to maximize the efficiency and clinical effectiveness of the system [70].

With enterprise PACS, the patient becomes the main focus of the operation and a single identifier (e.g. patient name or ID) is sufficient for any healthcare provider in the enterprise to retrieve the patient's comprehensive record [4]. At the enterprise level many hospitals and clinics put the emphasis on sharing enterprise integrated resources and streamlining daily (clinical) operations. In doing so, it is not necessary for all parties to have specialized services like radiology, since these kinds of services can be shared among all the entities in the enterprise through, for instance, teleradiology [10]. 
Wetering, R. van de, Batenburg, R. A PACS maturity model: a systematic meta-analytic review on maturation and evolvability of PACS in the hospital enterprise. International Journal of Medical Informatics: 2009 7062 127-140

In such an environment, images can be managed as an integral part of the EPR, so that clinicians will be able to use the same EPR front end to access images without having to learn new tools and user interfaces and use consultation and conferencing capabilities. This process improves the quality of medical treatments and care [13].

At such a development stage conventional hospital information systems, radiology information systems or other IS/IT are no longer sufficient, but the electronic patient record will prevail. Currently, the majority of the EPRs are not fully effective since they have not incorporated images. See also [71] and [72]. With the EPR the patient's record goes with the patient and information can easily be retrieved across different systems within the enterprise. Enterprise PACS should therefore have a direct connection with a central EPR server in order to distribute images and related data in a timely manner to a healthcare provider at the proper time and location [10]. An EPR requires a longitudinal, interoperable patient-specific record of all relevant imaging data, diagnostic reports and numerous other data resources, including several legacy systems [44]. Successful adoption and integration of these systems into the hospital enterprise is a considerable task.

A maturity-based typology in this context is provided by Whittick and Gill [44], who proposed a digital imaging electronic health record progression (DI/EHR) framework consisting of seven stages and several domains that constitute (DI/EHR). It describes the progression from printed film towards crossjurisdictional DI/EHR using several intermediary stages. The stages are:

1. Film/paper;

2. Modality storage/CD;

3. Dept. PACS/RIS;

4. Organization PACS/RIS;

5. Multi-organization shared PACS;

6. Regional shared DI-r;

7. Jurisdictional DI/EHR.

The stages are intended to express where a specific organization or jurisdiction may be. Moreover, Whittick does not suggest that organizations must progress through the stages sequentially. Although the model proposes several stages of progression for PACS, they are not all related to the maturity of PACS processes and their application within the hospital enterprise.

\section{THE PACS MATURITY MODEL (PMM)}

In this section, we build upon the results of the previous literature review concerning PACS maturity and evolvability, in order to construct a framework for maturity levels for PACS in the hospital enterprise. Following the meta-analytic approach, five levels of PACS maturity can be extracted from original resources:

\section{PACS infrastructure}

The initial level can be described as the basic and unstructured implementation and usage of image acquisition, storage, distribution and display. At this maturity level many technical and organizational problems arise with PACS owing to the lack of implemented standards in physical interfaces with the radiological sources, image and transfer formats and the dramatic changes that would result from PACS implementations [57].

II. PACS process

At the second maturity level most of the initial pitfalls have been covered by second generation PACS implementations [58], [59] and [60]. The focus of the "PACS process" maturity level is on effective process redesign, optimizing manual processes in radiology and initiating transparent PACS processes outside radiology. The focus, however, at this maturity level, is still only on medical images and is therefore restrictive in managing (hospital) workflows. The transition to filmless operation alone results in a relatively small gain in productivity if it is not accompanied by a redesign of the basic departmental workflow. This requires a high level of integration of the various imaging information systems and HIS and RIS [61].

III. Clinical process capability

The "clinical process capability" maturity level is represented by the evolution of PACS towards a system that can handle workflow and patient management [42], hospital-wide PACS distribution, communication and image-based clinical action. The evolution to this level requires important alterations in terms of PACS 
Wetering, R. van de, Batenburg, R. A PACS maturity model: a systematic meta-analytic review on maturatiog and evolvability of PACS in the hospital enterprise. International Journal of Medical Informatics: 2009 106 127-140

processes, extending the scope beyond imaging data and the level of integration of health information systems like HIS, RIS and PACS. At this level the clinical applicability of PACS begins to pay off by providing the imaging and associated (medical) documentation to clinicians, operating theatres, outpatient clinics and in some cases even outside the boundaries of the hospital.

IV. Integrated managed innovation

The fourth level of maturity is "integrated managed innovation" which can be characterized by the initial integration of PACS into the EPR and cross-enterprise exchange of digital imaging data and supporting documentation. This maturity level goes beyond clinical process capabilities with the adoption of emerging technologies like computer-assisted readings (CAR) and computer-aided diagnosis (CAD), resulting in decision support for clinical PACS usage. At this maturity level integrated PACS solutions are also applied for statistical information [38], intelligent data-mining purposes and quantitative control mechanisms [10]. Basically, the "integrated managed innovation" maturity level forms a bridge between the optimization of internal clinical PACS processes and the wider adoption within an EPR and enterprise PACS chain(s).

V. Optimized enterprise PACS chain

The final maturity level is the "optimized enterprise PACS chain". At enterprise level, and with PACS fully integrated into the ePR, the system can be maximized for efficiency purposes and clinical effectiveness [70]. Key process characteristics at this development stage include the following: large system integrations, PACS and web-based technology, see also [53], image distribution though web-based ePR [10]. Moreover, at this level the adoption within the wider EPR and healthcare facility integration is continually optimized and the operational improvements yield process innovations and overall efficiencies in the continuum of the patient-care delivery process.

A structured overview of the different PACS maturity levels with the associated PACS process focus and used references can be found in the Appendix A.

If we summarize the PACS maturity levels, together with the associated process focus and the relationship between the distinct levels of maturity, and depict this as a figure, the following maturity model appears (Fig. 2).

\section{[FIGURE 2]}

With the progression towards the highest PACS maturity level, the operational (workflow) efficiency, ITintegration, and effective and qualitative care using PACS technology expand. This is because of effective process redesign and optimization of underlying clinical processes and workflows, and the integration of PACS within a larger hospital enterprise (strategy). It goes without saying that this is not an easy task to achieve and it takes time to evolve.

With this progression, the level of retrieving more timely and accurate information for clinicians, physicians and hospital management increases as well. The process execution across the continuum of care improves patient care, allowing for real-time diagnosis, decision-support, inter-disciplinary processes, integration within the EPR, (intelligent) data-mining activities, continuous clinical optimization and so on. It should be noted, however, that high quality service, efficiency and clinical effectiveness of the PACS system can only be realized if an EPR is integrated with PACS. This integration enables a consistent working environment for clinicians, nurses and management and the opportunities for (e)consultation, for instance, teleconferencing [70] and [71].

If we look thoroughly at Fig. 2, we can also trace the three PACS trends that were identified in Section 4: (1) radiological and hospital-wide process improvements; (2) integration optimization and innovation; and (3) enterprise PACS and the electronic patient record (EPR). These three PACS trends overlap the maturity levels since they have a broader scope than just one distinct level of maturity. Trend 1 overlaps maturity levels 1,2 and a part of maturity level 3. The integration optimization and innovation trend overlaps maturity levels at part of level 2, 3 and part of level 4. The final PACS trend encloses maturity levels 4 and 5.

In some cases, we could use the retrieved studies to define a certain maturity level on a one-to-one basis. Other studies, however, addressed PACS process for several maturity levels. For this reason, some references are allocated to multiple maturity levels and can be seen in the appendix for an extensive overview. 
Wetering, R. van de, Batenburg, R. A PACS maturity model: a systematic meta-analytic review on maturatiog
and evolvability of PACS in the hospital enterprise. International Journal of Medical Informatics: 2009, 127-140

\section{DISCUSSION AND CONCLUSION}

There is an emerging need to evaluate PACS implementations as healthcare providers are facing both growing demand for improved care and higher expectations of service delivery [73]. Hospitals are pressured not only to spend money wisely, but also to demonstrate that it was wisely spent [74].

PACS is a system that is designed to streamline operations through the entire patient-care delivery system that is expected to make a significant difference in terms of throughput and clinical action as well. Earlier, Van de Wetering et al. [22] argued that theories on Business/IT-alignment, organizational fit and adoption of IS/IT can help to understand why certain key elements in clinical practice have not been realized.

Through a systematic review of PACS literature on maturity, and the construction of a maturity model for PACS using a meta-analytic approach, a first step has been taken towards an integrated framework for PACS alignment and implementation. It enables hospitals to quantify PACS maturity and relate this to PACS deployment performances in the hospital enterprise. We used a qualitative meta-analysis approach for the review and analysis of 34 selected scientific papers on PACS. This meta-analytic approach allowed us to structure the PACS literature in a new way and hence provide a basis for new theorization, i.e. the proposed maturity model. In this paper, it is actually demonstrated that PACS maturity levels can be extracted from scientific sources, and five maturity levels can be identified: (1) ad hoc PACS infrastructure; (2) PACS process; (3) clinical process capability; (4) integrated managed innovation; and (5) optimized PACS enterprise chain.

With the progression from one PACS maturity level to the highest level, the operational (workflow) efficiency, IT-integration, and effective and qualitative care using PACS technology expand so that process innovation and adoption within the wider EPRs and overall efficiencies in the continuum of the patient-care delivery process can be realized.

Although our definition of the five levels of PACS maturity is based on extensive literature study and analysis, in practice the five levels might not be fully exclusive or deterministic. Still, our five-level model is consistently based on a meta-analysis using a clustered process focus on communality, interrelationship and stage of maturity. Like in any type of modelling, we had to balance between recognizing the details of practice and complying the need for overview and limitation. Based on the existing body of knowledge and a rigour theoretical approach, we believe that the five levels defined for our PACS maturity model is optimal from both a scientific and practical perspective.

Several final comments can be made concerning our methodology. Although extensive, the literature search we performed concerns a single point of time (i.e. as of Winter, 2007). Obviously, new literature results and insight are being published with a rapid pace. It would therefore be an interesting elaboration to perform this literature research in later years. A result might be that the model needs to be extended. Also, we did not take into account the variety in quality and impact of the queried publications. In theory, it would be possible to cluster publications into a group of influential and often referenced papers, and papers that have a low impact and reference rate. Doing so, it can explored whether weighting of the reviewed papers change the results of this study.

A third and final discussion point concerns the structuring and replication of our qualitative meta-analysis, including the literature review and maturity model construction. In order to formalize the analyses we executed during all steps, one can think of explicitly modelling these, and associated deliverables through for instance - using a method engineering approach [75]

To the best of our knowledge, this paper is the first of its kind to review the maturity and evolvability concepts of PACS within hospitals and turn these into a maturity model. The proposed model is valuable for researchers as well as consultants and medical practitioners. It can be applied as a valuable tool for organizational assessments, monitoring and benchmarking purposes. Hence, the proposed PACS maturity model (PMM) contributes to an integral alignment model for PACS technology. By assessment of hospitals with the PMM, the model can be empirically validated. This results in data that can be used for benchmarking purposes. It should be noted, however, that the PMM does not provide a roadmap or blueprint on how to evolve through the levels of maturity. For this purpose, further research is required in order to explore what is needed to actually evolve through the different maturity levels. For each maturity level and the transformation from a certain level to the next one, a set of measurements can be defined. Although this is not elaborated in this paper, we suggest to organize these measurements into projects that take into account the risks involved, investment costs, critical success factors and benefits. In addition, an 
Wetering, R. van de, Batenburg, R. A PACS maturity model: a systematic meta-analytic review on maturatiog and evolvability of PACS in the hospital enterprise. International Journal of Medical Informatics: 2009 1062 127-140

alignment perspective [24] can be applied in managing similarities, overlap and synergy between the improvement projects.

As an example, our maturity model defines that improvements in operational efficiencies, IT-integration and effective and qualitative care can be achieved by integrating PACS within an EPR (see Fig. 2, maturity level 4: integrated managed innovation). This can be organized accordingly by a multidisciplinary project team that take into account both technological and organizational implications of such an improvement step.

A framework that elaborates such a practical and multidisciplinary alignment approach for PACS deployment is currently under investigation.

\section{CONTRIBUTIONS OF THE AUTHORS}

The authors initiated the idea to review PACS literature on maturity and evolvability and develop a PACS maturity model based on a qualitative meta-analysis. Both authors made substantial contributions to the design, acquisition of data and the subsequent analysis and interpretation of this study. Moreover, both authors revised the initial draft critically and approved the final version.

\section{SUMMARY POINTS}

What was known before the study:

- PACS and diagnostics imaging networks have been developed over a long period of time.

- PACS implementations range from simple IS/IT used for digitizing images to enterprise-wide image management systems and integrated workflow systems that streamline all operations throughout the entire patient-care delivery process.

- Literature on maturity and evolvability is fragmented.

\section{What this study has added:}

- A systematic literature review on 34 papers that sets out theoretical and empirical contributions on PACS maturity and evolvability.

- The identification of three development trends of PACS maturity and evolvability.

- The identification of five levels of PACS maturity that can be extracted from original resources using a qualitative meta-analysis approach.

- The development of a PACS maturity model (PMM) that can be applied for organizational assessments, monitoring and benchmarking purposes.

\section{REFERENCES}

[1] A. Hasman, Challenges for medical informatics in the 21st century, International Journal of Medical Informatics 44 (1997), pp. 1-7.

[2] H.K. Huang, Medical imaging informatics research and development trends-an editorial, Computerized Medical Imaging and Graphics 29 (2005), pp. 91-93.

[3] R.M. Mullner and K. Chung, Current issues in health care informatics, Journal of Medical Systems 30 (1) (2006), pp. 1-2.

[4] H.K. Huang, Enterprise PACS and image distribution, Computerized Medical Imaging and Graphics 27 (2003), pp. 241-253.

[5] E. Samei, J.A. Seibert, K. Andriole, A. Badano, J. Crawford and B. Reiner et al., AAPM/RSNA tutorial on equipment selection: PACS equipment overview. General guidelines for purchasing and acceptance testing of PACS equipment, Radio Graphics 24 (2004), pp. 313-334.

[6] D. Caramella, Is PACS research and development still necessary?, International Congress Series 1281 (2005), pp. 11-14.

[7] B. Kari, A.R. Mester, Z. Gyofi, B. Mihalik, Z. Hegyi and Z. Tarjan et al., Clinical evaluation of multimodality image archival and communication system in combination of WEB based teleradiology, International Congress Series 1281 (2005), pp. 974-979.

[8] M. Hood and H. Scott, Introduction to picture archive and communication systems, Radiology Nursing 25 (3) (2006), pp. 69-74.

[9] H.K. Huang, PACS is only in the beginning of being used as a clinical research tool, The 24th International EuroPACS Conference Trondheim, Norway (2006), pp. 1-10. 
Wetering, R. van de, Batenburg, R. A PACS maturity model: a systematic meta-analytic review on maturation and evolvability of PACS in the hospital enterprise. International Journal of Medical Informatics: 2009 706 . 127-140

[10] H.K. Huang, PACS and Imaging Informatics: Basic Principles and Applications, John Wiley \& Sons., Inc., Hoboken, New Jersey (2004).

[11] D. Anderson, K. Flynn, Picture Archiving and Communication Systems: A Systematic Review of Published Studies of Diagnostic Accuracy, Radiology Work Processes, Outcomes of Care, and Cost. Technology Assessment Program. 1997; Report No. 5.

[12] H.K. Huang, Some historical remarks on picture archiving and communication systems, Computerized Medical Imaging and Graphics 27 (2003), pp. 93-99. [13] N.-T. Cheung, A. Lam, W. Chan and J. Kong, Integrating images into the electronic patient record of the hospital authority of Hong Kong, Computerized Medical Imaging and Graphics 29 (2005), pp. 137-142.

[14] R.L. Nolan, Managing the computer resource: a stage hypothesis, Communications of the Association for Computing Machinery 16 (7) (1973), pp. 399-405.

[15] C.F. Gibson and R.L. Nolan, Managing the four stages of EDP growth, Harvard Business Review 52 (1) (1974), pp. 76-88.

[16] I. Benbasat, A.S. Dexter and R.W. Mantha, Impact of organizational maturity on information skill needs, MIS Quartely 4 (1) (1980), pp. 21-34.

[17] M.J. Earl, Management Strategies for Information Technologies, Prentice Hall, New Jersey (1989).

[18] R. Galliers and S.K. Somogyi, From data processing to strategic information systems-a historical perspective. In: S.K. Galliers RaS, Editor, Towards Strategic Information Systems, Bacus Press, Cambridge, MA (1987), pp. 5-25.

[19] R.D. Galliers and A.R. Sutherland, Information systems management and strategy formulation: the 'stages of growth' model revisited, Journal of Information Systems 1 (2) (1991), pp. 89-114.

[20] J.L. King and K.L. Kraemer, Evolution and organizational information systems: an assessment of Nolan's stage model, Communications of the Association for Computing Machinery 27 (5) (1984), pp. 466-475.

[21] J.J. Jiang, G. Klein and M. Shepherd, The materiality of information system planning maturity to project performance, Journal of the Association for Information Systems (2001), p. 2 (Article 5, September).

[22] R. Van de Wetering, R. Batenburg, J. Versendaal, R. Lederman and L. Firth, A balanced evaluation perspective: picture archiving and communication system impacts on hospital workflow, Journal of Digital Imaging 19 (Suppl. 1) (2006), pp. 10-17.

[23] J. Luftman, Assessing business-IT alignment maturity, Communications of the Association for Information Systems 4 (14) (2000), pp. 1-49.

[24] W.J. Scheper, Business IT Alignment: Solution for the Productivity Paradox (in Dutch), Deloitte \& Touche, The Netherlands (2002).

[25] U. Bick and H. Lenzen, PACS: the silent revolution, European Radiology 9 (1999), pp. 1152-1160.

[26] K. Doi, Diagnostic imaging over the last 50 years: research and development in medical imaging science and technology, Physics in Medicine and Biology 51 (2006), pp. 5-27.

[27] D.B. Bandon, P. Trolliard, A. Garcia, C. Lovis, A. Geissbühler and J.-P. Vallée, Building an enterprisewide PACS for all diagnostic images, International Congress Series 1268 (2004), pp. 279-284.

[28] IHE. Integrating the Healthcare Enterprise. 2007 [cited 200711 November, 03:15 PM]; Available from: http://www.ihe.net/.

[29] O. Ratib and A. Rosset, Can PACS benefit from general consumer communication tools, International Congress Series 1281 (2005), pp. 948-953.

[30] E.M. Rogers, Diffusion of Innovations, Free Press, New York (1995).

[31] Team CPD. CMMI for systems engineering/software engineering/integrated product and process development/supplier sourcing, version 1.1 continuous representation. Technical Report CMU/SEI-2002TR-011, ESC-TR-2002-011, Software Engineering Institute, Carnegie Mellon University, Pittsburgh, PA, USA. 2000.

[32] T. De Bruin and M. Rosemann, Understanding the main phases of developing a maturity assessment model, Proceedings of the16th Australasian Conference on Information Systems Sydney (2005).

[33] D. Galin and M. Avrahami, Do SQA programs work -CMM works. A meta analysis. In: R. Amir Tomer and S.R. Schach, Editors, Proceedings of the International Conference on Software-Science, Technology \& Engineering (SwSTE'05), USA IEEE Computer Society Press, Washington, DC (2005), pp. 95-100.

[34] C. Holland and B. Light, A stage maturity model for enterprise resource planning systems, Data Base for Advances in Information Systems 32 (2) (2001), pp. 34-45.

[35] J. Damsgaard and R. Scheepers, Managing the crises in intranet implementation: a stage model, Information Systems Journal 10 (2) (2000), pp. 131-149.

[36] R. Haux, Health information systems-past, present, future, International journal of Medical Informatics 75 (2005), pp. 268-281. 
Wetering, R. van de, Batenburg, R. A PACS maturity model: a systematic meta-analytic review on maturation and evolvability of PACS in the hospital enterprise. International Journal of Medical Informatics: 2009 106 127-140

[37] L.H. Vogel, Finding value from IT investments: exploring the elusive ROI in healthcare, Journal of Healthcare Information Management 17 (4) (2003), pp. 20-28.

[38] Azevedo-Marques PMd, E.C. Caritá, A.A. Benedicto and P.R. Sanches, Integrating RIS/PACS: the Web-based Solution at University Hospital of Ribeirao, Brazil, Journal of Digital Imaging 17 (3) (2004), pp. 226-233.

[39] O. Ratib, F. Terrier and J. Scherrer, Evolution of PACS concept in the hospital enterprise, RBM 18 (5) (1996), pp. 112-121.

[40] S.S. Buchbinder, Breast imaging, computer-aided detection, and computer-assisted classification. In: K.J. Dreyer, D.S. Hirschorn, J.H. Thrall and A. Metha, Editors, PACS: A Guide to the Digital Revolution, Springer, New York (2006), pp. 433-446.

[41] G.J. Harris, Three dimensional imaging in radiology. In: K.J. Dreyer, D.S. Hirschorn, J.H. Thrall and A. Metha, Editors, PACS: A Guide to the Digital Revolution, Springer, New York (2006), pp. 447-466.

[42] G.D. Laet, J. Naudts and J. Vandevivere, Workflow in nuclear medicine, Computerized Medical Imaging and Graphics 25 (2001), pp. 195-199.

[43] E.L. Siegel, B. Reiner and N. Knight, Reengineering workflow: the radiologist's perspective. In: K.J. Dreyer, D.S. Hirschorn, J.H. Thrall and A. Metha, Editors, PACS: A Guide to the Digital Revolution, Springer, New York (2006), pp. 98-123.

[44] D. Whittick and S. Gill, Diagnostic imaging electronic health record (DI/EHR) challenges-strategy and planning perspective, The 24th International EuroPACS Conference Trondheim, Norway (2006), pp. 1-9.

[45] G.W. Noblit and R.D. Hare, Meta-ethnography: synthesizing qualitative studies, Sage, Newbury Park, CA (1988).

[46] B.L. Paterson, S. Thorne, C. Canam and C. Jillings, Meta-study of qualitative health research: A practical guide to meta-analysis and meta-synthesis, Sage, Thousand Oaks, CA (2001).

[47] In: H.M. Cooper and J.J. Lindsay, Editors, Research Synthesis and Meta-Analysis, Sage, Thousand Oakes: CA (1998).

[48] T. Bondas and E.O.C. Hall, Challenges in approaching metasynthesis research, Qualitative Health Research 17 (1) (2007), pp. 113-121.

[49] J. McCormick, P. Rodney and C. Varcoe, Reinterpretations across studies: an approach to metaanalysis, Qualitative Health Research 13 (7) (2003), pp. 933-944.

[50] S. Thorne, B. Paterson, S. Acorn, C. Canam, G. Joachim and C. Jillings, Chronic illness experience: insights from a metastudy, Qualitative Health Research 12 (4) (2002), pp. 437-452.

[51] S. Zhao, Metatheory, metamethod, qualitative meta-analysis: what, why, and how?, Sociological Perspectives 34 (4) (1991), pp. 377-390. View Record in Scopus | Cited By in Scopus (29)

[52] M. Sandelowski, S. Docherty and C. Emden, Qualitative metasynthesis: Issues and techniques, Research in Nursing \& Health 20 (1997), pp. 365-371.

[53] J. Zhang, J. Sun and J.N. Stahl, PACS and web-based image distribution and display, Computerized Medical Imaging and Graphics 27 (2003), pp. 197-206.

[54] M.D. Ralston, R.M. Coleman, D.M. Beaulieu, K. Scrutchfield and T. Perkins, Progress towards paperless radiology in the digital environment: planning, implementing, and benefits, Journal of Digital Imaging 17 (2) (2004), pp. 134-143.

[55] T. Warfel and P.J. Chang, Integrating dictation with PACS to eliminate paper, Journal of Digital Imaging 17 (1) (2004), pp. 37-44.

[56] M. Li, D. Wilson, M. Wong and A. Xthona, The evolution of display technologies in PACS applications, Computerized Medical Imaging and Graphics 27 (2003), pp. 175-184.

[57] M. Osteaux, R. Broeck Vd, F. Verhelle and Jd. Mey, Picture archiving and communication system (PACS): a progressive approach with small systems, European Journal of Radiology 22 (1996), pp. 166174.

[58] B. Reiner and E.L. Siegel, Workflow optimization: current trends and future directions, Journal of Digital Imaging. 15 (3) (2002), pp. 141-152.

[59] B. Reiner and E.L. Siegel, The work flow imperative, The Journal of Imaging Technology Management (2003) February.

[60] B. Reiner, E.L. Siegel and K. Siddiqui, Evolution of the digital revolution: a radiologist perspective, Journal of Digital Imaging. 16 (4) (2003), pp. 324-330. [61] E.L. Siegel and B. Reiner, Work flow redesign: the key to success when using PACS, Journal of Digital Imaging. 16 (1) (2003), pp. 164-168.

[62] B. Crowe and L. Sim, An assessment of the effect of the introduction of a PACS and RIS on clinical decision making and patient management at Princess Alexandra Hospital Brisbane, Australia, International Congress Series 1281 (2005), pp. 964-967.

[63] H. Fu, Z. Jin, J. Dai, K. Chen, T. Wang and T. Li et al., Picture archiving and communication system in China: the development, problem, and integrating strategy with IHE, International Congress Series 1256 (2003), pp. 915-923. 
Wetering, R. van de, Batenburg, R. A PACS maturity model: a systematic meta-analytic review on maturation and evolvability of PACS in the hospital enterprise. International Journal of Medical Informatics: 2009 1062 127-140

[64] C.C.T. Lim, G.L. Yang, W.L. Nowinski and F. Hui, Medical image recourse center-making electronic teaching files from PACS, Journal of Digital Imaging 16 (4) (2003), pp. 331-336.

[65] C.-H. Hsiao, T.-C. Hsu, J.N. Chang, S.J.H. Yang, S.-T. Young and W.C. Chu, Developing a medical image content repository for e-learning, Journal of Digital Imaging 19 (3) (2006), pp. 207-215.

[66] S. Erberich, PACS-based functional magnetic resonance imaging, Computerized Medical Imaging and Graphics 27 (2003), pp. 229-240.

[67] E. Peitka, S. Pospiech-Kurkowska, A. Gertych and F. Cao, Integration of computer assisted bone age assessment with clinical PACS, Computerized Medical Imaging and Graphics 27 (2003), pp. 217-228.

[68] T. Kalinske, H. Hofmann, D.-S. Franke and A. Roessner, Digital imaging and electronic patient records in pathology using an integrated department information system with PACS, Pathology Research and Practice 198 (2002), pp. 679-684.

[69] O. Ratib, M. Swiernik and J.M. McCoy, From PACS to integrated EMR, Computerized Medical Imaging and Graphics 27 (2003), pp. 207-215.

[70] E.L. Siegel and B. Reiner, Filmless radiology at the Baltimore VA Medical Center: a 9 year retrospective, Computerized Medical Imaging and Graphics 27 (2003), pp. 101-109.

[71] H. Munch, U. Engelmann, A. Schroeter and H.P. Meinzer, Web-based distribution of radiological images from PACS to EPR, International Congress Series 1256 (2003), pp. 873-879.

[72] H. Munch, U. Engelmann, A. Schröter and H.P. Meinzer, The integration of medical images with the electronic patient record and their web-based distribution, Academic Radiology 11 (2004), pp. 661-668.

[73] J. Ahovuo, O. Tolkki, N. Fyhr and J. Kujala, Process oriented organisation in the regional PACS environment, EuroPACS-MIR 2004 in the Enlarged Europe (2004), pp. 481-484.

[74] J.C. Wyatt and S.M. Wyatt, When and how to evaluate health information systems?, International Journal of Medical Informatics 69 (2003), pp. 251-259.

[75] In: J. Ralyté, S. Brinkkemper and B. Henderson-Sellers, Editors, Situational Method Engineering: Fundamentals and Experiences, Springer, New York (2007).

[76] B. Reiner, E.L. Siegel, Guest Editors. The cutting edge: strategies to enhance radiologist workflow in a filmless/paperless imaging department. Journal of Digital Imaging 2002; 15(3):178-190.

[77] E.L. Siegel, Z. Protopapas, B. Reiner, S. Pomerantz, E.W. Cameron and E. Pickar, A prospective evaluation of the impact of filmless operation of the Baltimore VA Medical Center, RBM 18 (5) (1996), pp. 149-152.

[78] E.L. Siegel and B. Reiner, Radiology reading room design: the next generation, Applied Radiology 31 (3) (2002), pp. 11-16. Abstract | View Record in Scopus / Cited By in Scopus (9)

[79] C. Bozec, E. Zapletal, M.-C. Jaulent, D. Heudes and P. Degoulet, Towards content-based image retrieval in HIS-integrated PACS, Proceedings of the Annual Symposium of the American Society for Medical Informatics (AMIA); 2000 Los Angeles, CA, USA (2000), pp. 477-481.

[80] D. Bandon, C. Lovis, A. Geissbühler and J.-P. Valleé, Enterprise-wide PACS: beyond radiology, an architecture to manage all medical images, Academic Radiology 12 (2005), pp. 1000-1009. 
Wetering, R. van de, Batenburg, R. A PACS maturity model: a systematic meta-analytic review on maturation and evolvability of PACS in the hospital enterprise. International Journal of Medical Informatics: 2009, 700 127-140

\section{FIGURES, TABLES AND APPENDIX}

Fig. 1 - PACS usage in Europe.

\section{Proportion using PACS}

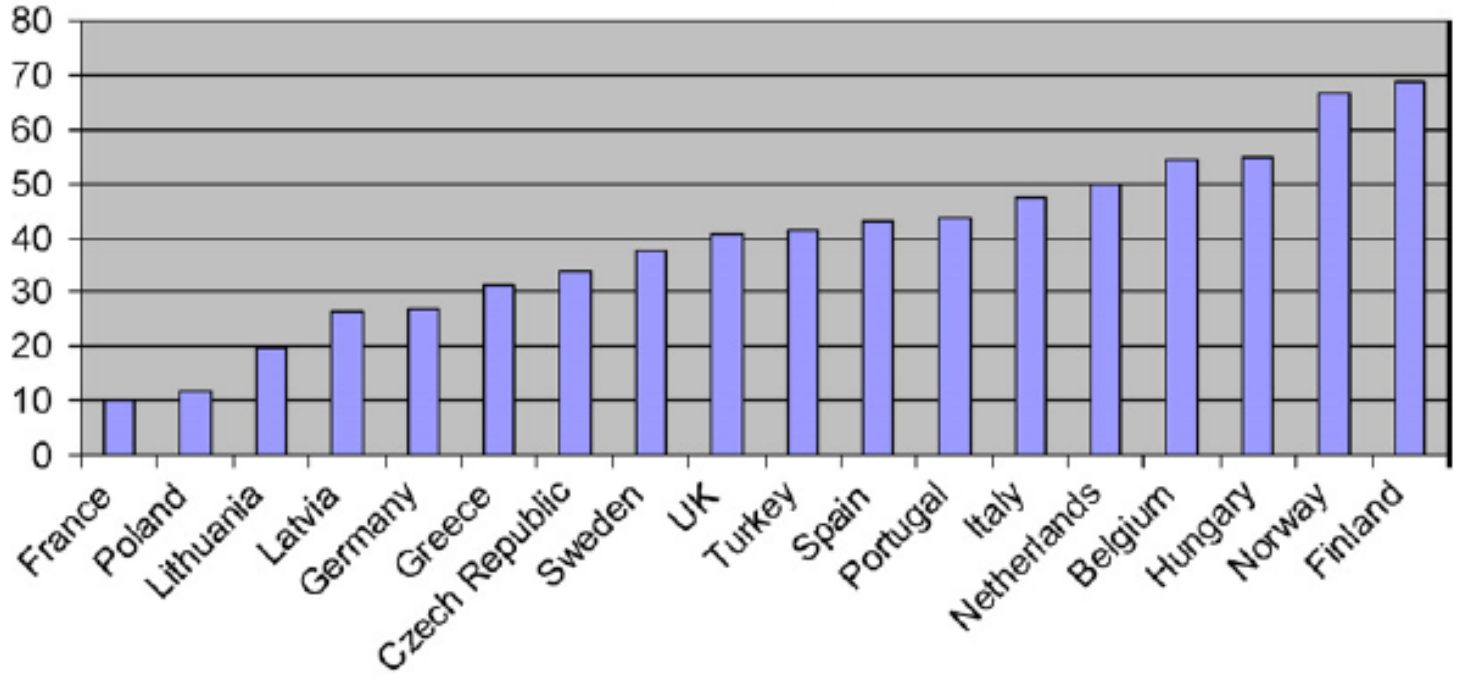

\section{Table 1 - Maturity levels of the CMMI}

\begin{tabular}{|c|c|}
\hline Maturity stage & Clarification \\
\hline Initial & $\begin{array}{l}\text { - Carry out work on an ad hoc basis } \\
\text { - Formal processes unclear } \\
\text { - Poor control } \\
\text { - Effort of a few enterprising individuals } \\
\text { instead of the whole organization }\end{array}$ \\
\hline Repeatable & $\begin{array}{l}\text { - Depend on policies for managing a } \\
\text { software process and measures } \\
\text { - Processes of the organizations stay } \\
\text { institutionalized through experience } \\
\text { instead of detailed procedures }\end{array}$ \\
\hline Defined & $\begin{array}{l}\text { - Engineering activities and processes of } \\
\text { management are formally defined } \\
\text { - Newer methods and tools can be } \\
\text { added }\end{array}$ \\
\hline Managed & $\begin{array}{l}\text { - Detailed measures of the software } \\
\text { process and product quality are collected } \\
\text { - Setting quantitative goals } \\
\text { - New sets of tools are } \\
\text { added/adjustments are made to existing } \\
\text { processes }\end{array}$ \\
\hline Optimizing & $\begin{array}{l}\text { - Focus on continuous process } \\
\text { improvement } \\
\text { - Instead of correcting defects, a firm } \\
\text { stalls future defects and addresses the } \\
\text { key to those defects by planning advance }\end{array}$ \\
\hline
\end{tabular}



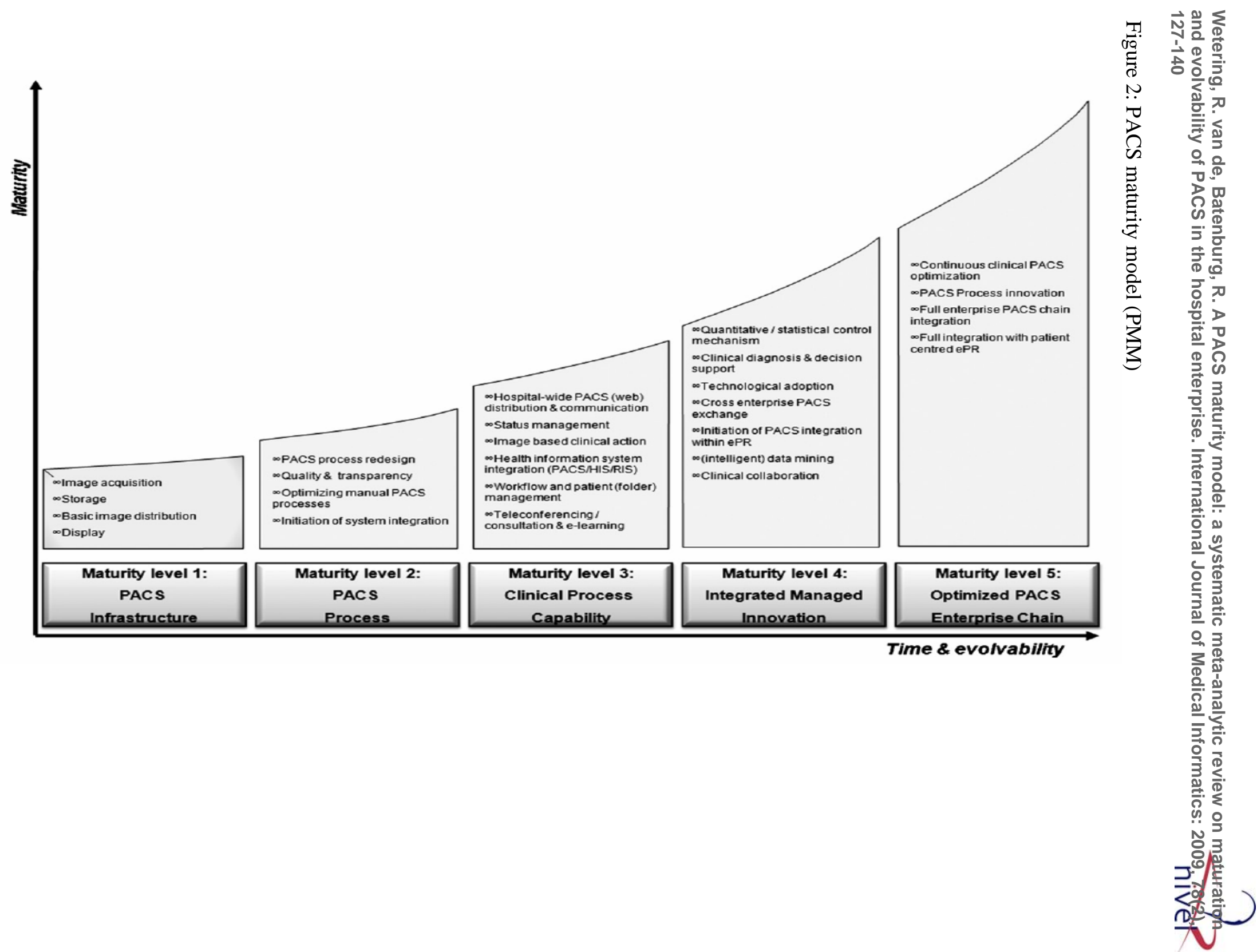
Wetering, R. van de, Batenburg, R. A PACS maturity model: a systematic meta-analytic review on maturation and evolvability of PACS in the hospital enterprise. International Journal of Medical Informatics: 2009, 70t2 127-140

\section{Appendix A. PACS maturity levels}

\begin{tabular}{|c|c|c|}
\hline PACS maturity level & PACS process focus & Used references \\
\hline (1) PACS infrastructure $\square$ & $\begin{array}{l}\text { Basic and unstructured usage of the following } \\
\text { processes: } \\
\text { - Image acquisition process } \\
\text { - Storage process } \\
\text { - Image distribution process } \\
\text { - Display process }\end{array}$ & $\begin{array}{l}\sqrt{ }[57] \\
\sqrt{ }[25] \\
\sqrt{ }[56]\end{array}$ \\
\hline (2) PACS process $\square \square$ & $\begin{array}{l}\text { The process focus of the "PACS process" maturity } \\
\text { level is on: } \\
\text { - PACS process redesign } \\
\text { - Quality and transparency } \\
\text { - Optimizing manual PACS processes } \\
\text { - Initiation of system integration } \\
\text { - Qualitative measurements }\end{array}$ & $\begin{array}{l}\sqrt{ }[63] \\
\sqrt{ }[59-61,70,76-78] \\
\sqrt{ }[56] \\
\sqrt{ }[39] \\
\sqrt{ }[54]\end{array}$ \\
\hline (3) Clinical process capability $\square \square$ & $\begin{array}{l}\text { The process focus of the "Clinical Process } \\
\text { Capability" maturity level is on: } \\
\text { - Hospital-wide PACS (web) distribution and } \\
\text { communication } \\
\text { - Control/status management } \\
\text { - Patient (folder) management } \\
\text { - Image-based clinical action } \\
\text { - HIS/RIS/PACS (multimedia) integration } \\
\text { - Workflow management } \\
\text { - Teleconferencing/consultation and e-learning }\end{array}$ & $\begin{array}{l}\sqrt{ }[42] \\
\sqrt{ }[63] \\
\\
\sqrt{ }[9] \\
\sqrt{ }[64] \\
\sqrt{ }[62] \\
\sqrt{ }[56] \\
\sqrt{ }[7] \\
\sqrt{ }[79] \\
\sqrt{ }[53] \\
\sqrt{ }[38] \\
\sqrt{ }[65] \\
\sqrt{ }[9]\end{array}$ \\
\hline (4) Integrated managed innovation & $\begin{array}{l}\text { The process focus of the "Integrated managed } \\
\text { innovation" maturity level is on: } \\
\text { - Quantitative/statistical control mechanism } \\
\text { - Clinical diagnosis and decision support } \\
\text { - Technological adoption (e.g. CAD, IASS, FFDM, } \\
\text { bone age assessment) } \\
\text { - Cross-enterprise PACS exchange } \\
\text { - Initiation of PACS integration within EPR } \\
\text { - (intelligent) data-mining } \\
\text { - Clinical collaboration }\end{array}$ & $\begin{array}{l}\sqrt{ }[63] \\
\sqrt{ }[64] \\
\sqrt{ }[62] \\
\sqrt{ }[68] \\
\\
\sqrt{ }[64,65] \\
\sqrt{ }[69,80] \\
\sqrt{ }[4,13,53,71,72] \\
\sqrt{ }[8,44] \\
\sqrt{ }[10] \\
\sqrt{ }[38] \\
\sqrt{ }[7] \\
\sqrt{ }[80] \\
\sqrt{ }[67] \\
\sqrt{ }[66]\end{array}$ \\
\hline
\end{tabular}

(5) Optimized enterprise PACS chain

The process focus of the "Optimized Enterprise

PACS Chain" maturity level is on:

- Continuous clinical PACS optimization

- PACS Process innovation

- Full enterprise PACS chain integration

- Full integration with patient-centred EPR 